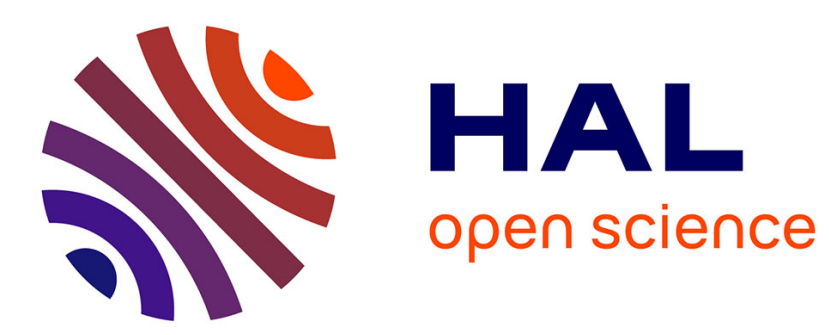

\title{
ANALYSIS OF SOLID-LIQUID INTERPHASE SPECTRA VIA CAUSAL TRANSFORMATION
}

\author{
W. Hansen, W. Abdou
}

\section{To cite this version:}

W. Hansen, W. Abdou. ANALYSIS OF SOLID-LIQUID INTERPHASE SPECTRA VIA CAUSAL TRANSFORMATION. Journal de Physique Colloques, 1977, 38 (C5), pp.C5-207-C5-214. 10.1051/jphyscol:1977527 . jpa-00217176

\section{HAL Id: jpa-00217176 https://hal.science/jpa-00217176}

Submitted on 1 Jan 1977

HAL is a multi-disciplinary open access archive for the deposit and dissemination of scientific research documents, whether they are published or not. The documents may come from teaching and research institutions in France or abroad, or from public or private research centers.
L'archive ouverte pluridisciplinaire HAL, est destinée au dépôt et à la diffusion de documents scientifiques de niveau recherche, publiés ou non, émanant des établissements d'enseignement et de recherche français ou étrangers, des laboratoires publics ou privés. 


\title{
ANALYSIS OF SOLID-LIQUID INTERPHASE SPECTRA VIA CAUSAL TRANSFORMATION
}

\author{
W. N. HANSEN and W. A. ABDOU \\ Utah State University, Logan, Utah 84322, U. S. A.
}

\begin{abstract}
Résumé. - Nous présentons un procédé pour le calcul du spectre d'absorption intrinsèque (i. e., l'indice de réfraction complexe, ou la constante diélectrique en fonction de la fréquence) pour des films minces ainsi que pour des couches adsorbées à partir des spectres de réflexion et de transmission, utilisant une transformation causale. Le film que nous étudions peut faire partie d'un milieu stratifié ayant plusieurs couches. Notre méthode se base pour l'utilisation de la causalité de la fonction $G(\omega)$ qui est définie par l'expression ln $\left(\hat{r}_{s}(\omega) / \hat{r}_{\mathrm{b}}(\omega)\right)$, où $\hat{r}_{\mathrm{s}}$ est le coefficient de réflexion de Fresnel pour l'échantillon et $\hat{r}_{\mathbf{b}}$ est le même coefficient si l'on imagine que l'échantillon n'adsorbe plus la radiation électromagnétique dans l'intervalle du spectre auquel nous nous intéressons.

Abstract. - Procedures are presented for calculating intrinsic absorption spectra (i. e., the complex refractive index or dielectric constant as a function of frequency) of thin films and adsorbed layers from reflection and transmission spectra by means of a causal transformation. The film being studied may be part of a multi-layer stratified medium. The basis of the method is the causality of the function $G(\omega)=\ln \left(\hat{r_{\mathrm{s}}}(\omega) / \hat{r_{\mathrm{b}}}(\omega)\right)$, where $\hat{r_{\mathrm{s}}}$ is the Fresnel reflection coefficient of the sample material and $\hat{r}_{\mathrm{D}}$ is the coefficient of the same sample after an imagined electromagnetic bleaching in the spectral range of interest.
\end{abstract}

1. Introduction. - In a previous paper [1] a new method was presented for obtaining intrinsic absorption spectra (i. e. index of refraction, $n$ and extinction coefficient, $k$, vs. wavelength) from reflection spectra of stratified media. A similar approach is valid for transmission spectra. The basis of the method is the proven causality of a special reflection function which we introduce, and the application to this function of the transformation given by Peterson and Knight [2], hereafter referred to as the PK transformation. The method has general applicability to reflection and transmission spectra of stratified media. Of course, the accuracy with which the intrinsic spectra are determined depends upon the information content of the raw spectrum used. This can in turn be optimised by the wise choice of experimental parameters used in taking the raw spectrum. For example, consider a highly reflecting metal surface with an adsorbed corrosion inhibiting organic layer that we wish to study by means of infrared spectroscopy. A reflection spectrum could be taken using radiation polarized perpendicular to the plane of incidence, $\perp$, and the resulting spectrum could be transformed, but it would yield little or no information about the organic film regardless of the angle of incidence chosen. If parallel polarization, $\|$, were used however, and at oblique incidence, both $n(\omega)$ and $k(\omega)$ could be obtained as a function of frequency, $\omega$. The reason is that $\perp$ or normal incident reflectance is insensitive to the presence of the layer.

The present paper is concerned with the application of the method to spectra of thin regions between bulk phases. It is particularly aimed at very thin adsorbed layers and other thin layers that may be hard to analyze otherwise. Examples are reflection and transmission spectra of the electrochemical interphase region. Reflection may be internal or external. Adsorbed films on thin layer metal or semiconductor electrodes are typical examples. Another example is bulk metal surface treated by immersion in some organic or aqueous solution and then removed and examined by reflection in the infrared.

The interphase regions need not be layers in the ordinary sense. They may comprise perturbed surface regions of a solid phase and complicated double layer structures of a bordering electrolyte. Even so, if the inhomogeneities are small compared to a wavelength of the light involved, and if the statistical average of microscopic polarizability varies only in the direction perpendicular to the interface, the composite is a stratified medium, and the relevant optical theory applies. The transformation theory applies just as well to field induced changes in the interphase as it does to a fixed classical thin film.

The basis of the general method is as follows. The contrived complex function of complex $\omega$

$$
G(\omega) \equiv \ln \left[\hat{r}_{\mathrm{s}}(\omega) / \hat{r}_{\mathrm{b}}(\omega)\right]=\frac{1}{2} \ln \left(R_{\mathrm{s}} / R_{\mathrm{b}}\right)+i\left(\theta_{\mathrm{s}}-\theta_{\mathrm{b}}\right)
$$

is causal, i. e., subject to the PK transformation. Here $\hat{r}$ refers to the fresnel reflection coefficient, « $\mathrm{s} »$ to the sample layer being studied, $« \mathrm{~b} »$ to the same sample 
imagined to be electromagnetically bleached in the frequency region of interest, or otherwise changed in special cases as in the case of electroreflectance. Reflec- tance is indicated by $R$ and phase angle by $\theta$. Letting $G(\omega) \equiv \operatorname{Re}(\omega)+i \operatorname{Im}(\omega) \equiv S(\omega)+i A(\omega)$, then the PK transformation is given by

$$
S\left(\omega_{0}\right)=-\frac{i}{2 \pi} \int_{-\infty}^{0} \mathrm{~d} t \mathrm{e}^{-i \omega_{0} t} \int_{-\infty}^{\infty} \mathrm{d} \omega \mathrm{e}^{i \omega t} A(\omega)+\frac{i}{2 \pi} \int_{0}^{\infty} \mathrm{d} t \mathrm{e}^{-i \omega_{0} t} \int_{-\infty}^{\infty} \mathrm{d} \omega \mathrm{e}^{i \omega t} A(\omega)
$$

and

$$
i A\left(\omega_{0}\right)=\frac{-1}{2 \pi} \int_{-\infty}^{0} \mathrm{~d} t \mathrm{e}^{-i \omega_{0} t} \int_{-\infty}^{\infty} \mathrm{d} \omega \mathrm{e}^{i \omega t} S(\omega)+\frac{1}{2 \pi} \int_{0}^{\infty} \mathrm{d} t \mathrm{e}^{-i \omega_{0} t} \int_{-\infty}^{\infty} \mathrm{d} \omega \mathrm{e}^{i \omega t} S(\omega)
$$

or in simpler form (see Appendix A)

$$
S\left(\omega_{0}\right)=-\frac{2}{\pi} \int_{0}^{\infty} \int_{0}^{\infty} A(\omega) \sin \omega t \cos \omega_{0} t \mathrm{~d} \omega \mathrm{d} t
$$

and

$$
A\left(\omega_{0}\right)=-\frac{2}{\pi} \int_{0}^{\infty} \int_{0}^{\infty} S(\omega) \cos \omega t \sin \omega_{0} t \mathrm{~d} \omega \mathrm{d} t
$$

Here $S(\omega)$ is symmetric in $\omega$ and $A(\omega)$ is antisymmetric.

The well known Kramers-Kronig transformation can be applied here [2] and is equivalent analytically, but not as actually carried out numerically by computer. The PK method is more efficient, and errors are easier to discover.

In this paper we first illustrate application of the method to the analysis of transmission spectra to get the intrinsic spectra of the sample. Liquid benzene is used as an example for detailed treatment, and the resulting spectrum of $n(\omega)$ is compared to literature values. The application to internal reflection spectra is then discussed. Internal reflection has certain advantages including high sensitivity, and the fact that it is usually easy to get $R_{\mathrm{s}} / R_{\mathrm{b}}$ from the raw reflectance spectrum. External reflectance is next analyzed. The special external reflection case of a film on a highly reflecting metal surface is discussed in detail because of its importance and ease of analysis.

2. Application to transmission spectra. - The transmittance, $T$, of a sample of thickness $h$ is given in terms of absorbance, $A$, as

$$
A_{\mathrm{T}} \equiv \log _{10} T^{-1} \equiv \log _{10}\left(I^{0} / I\right)=\frac{4 \pi h}{\lambda \ln 10} k
$$

where $I$ is radiation intensity, $h$ is thickness, $\hat{\lambda}$ is wavelength, and $k$ is the imaginary part of the complex refractive index $\hat{n}=n+i k$. The thickness is known or determined. Thus from good transmission data, $k$ is known directly. In actual practice, for high accuracy, $A_{T}$ is also measured with the sample replaced by a transparent phase of real refractive index similar to that of the bleached sample. Thus the actual measurement is of

$$
\Delta A_{\mathrm{T}}=A_{\mathrm{s}}-A_{\mathrm{b}}=\frac{4 \pi h}{\lambda \ln 10}\left(k_{\mathrm{s}}-k_{\mathrm{b}}\right)
$$

where « $s$ » and $\langle b$ » again refer to regular sample and electromagnetically bleached sample. The transparent phase used (liquid or solid) is taken as similar as needed to the bleached sample in the spectral region of interest.

Another scheme is to use an adjustable path length transmission cell. Here $\Delta A_{\mathrm{T}}$ is measured for a known increase in path length $\Delta h$. The same measurement is made with a transparent liquid of index close to that of the bleached sample. This latter $\Delta A_{\mathrm{r}}$ should be close to zero, but may give a small correction due to beam path changes. We now have, overall

$$
\Delta A_{\mathrm{T}}=\Delta A_{\mathrm{s}}-\Delta A_{\mathrm{b}}=\frac{4 \pi \Delta h}{\lambda \ln 10}\left(k_{\mathrm{s}}-k_{\mathrm{b}}\right)
$$

where it is assumed that small contributions to $\Delta A_{\mathrm{s}}$ and $\Delta A_{\mathrm{b}}$ from causes other than $k_{\mathrm{s}}$ and $k_{\mathrm{b}}$ will be equal and cancel. Actually, for very strong absorption bands where anomalous dispersion gives rise to large changes in coherent interference, even these procedures may not suffice for highest accuracy. For this case a better approach would be to use reflection techniques.

In eq. (7) and (8) $k_{\mathrm{b}}$ is essentially zero, but it is instructive to leave it in the equation. It is well known that $\hat{n}-1$ is causal and thus subject to PK transformation, i. e., it satisfies eq. (2)-(5) if we let $S(\omega) \equiv n-1$ and $A(\omega) \equiv k$. It is easily seen from these equations that the sum of causal functions is itself causal. Therefore, $\hat{n}_{\mathrm{s}}-\hat{n}_{\mathrm{b}}$ is causal. It was shown in reference (1) that $k_{\mathrm{b}}$ is essentially equal to $k_{\mathrm{s}}$ everywhere except in the region where the spectra are taken, and in this region it is essentially zero. Therefore $\operatorname{Im}\left(\hat{n}_{\mathrm{s}}-\hat{n}_{\mathrm{b}}\right) \equiv k_{\mathrm{s}}-k_{\mathrm{b}}$ is known at all frequencies. Therefore a PK transformation gives $\operatorname{Re}\left(\hat{n}_{\mathrm{s}}-\hat{n}_{\mathrm{b}}\right) \equiv\left(n_{\mathrm{s}}-n_{\mathrm{b}}\right)$ at all frequencies desired. To procede further we must have minor addi- 
tional information which is not contained in the spectral data and cannot be obtained from it no matter how clever we are. Auxiliary measurements (see Appendix B) can supply $n_{s}$ at two frequencies, usually on each side of the absorption band. This determines $n_{\mathrm{b}}$ at the same frequencies. Since $n_{\mathrm{b}}$ is nearly linear throughout the bleached region, $n_{\mathrm{s}}$ is easily calculated throughout the region of interest. Thus, from the transmission spectrum plus some minor information $n_{\mathrm{s}}(\omega)$ is determined throughout the region of interest.

To illustrate this procedure the transmission spectrum of benzene is used. Figure 1 is such a spectrum for

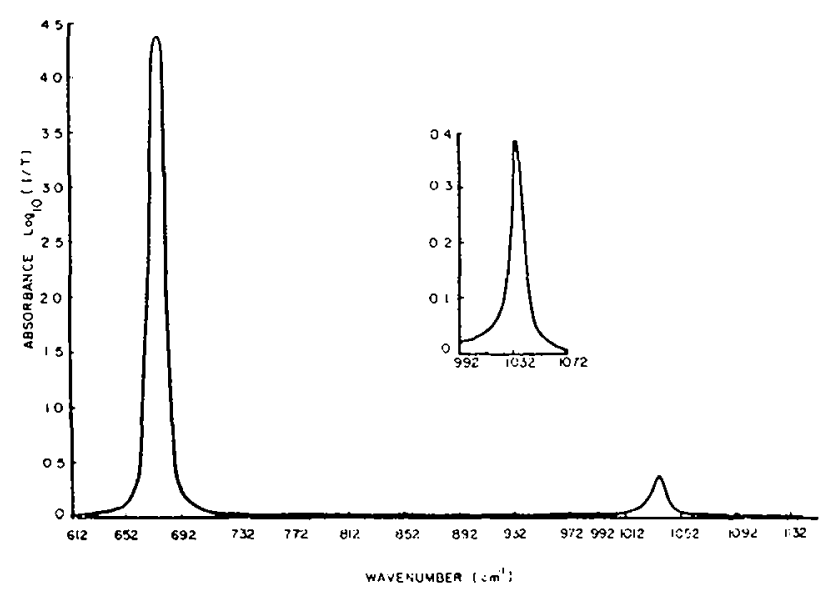

FIG. 1. - Transmission spectrum of a $10 \mu$ thick layer of liquid benzene.

a range including two main absorption bands. These bands were chosen because the optical constants have been determined carefully at each wavelength by internal reflection techniques by others [3]. The transmission data of figure 1 were transformed to get $n_{\mathrm{s}}(\omega)$. This $n$ is compared with the $\mathrm{n}$ determined by the reflection techniques. Results are shown in figure 2. The agreement is excellent and confirms not only our procedures but their data, obtained by methods independent

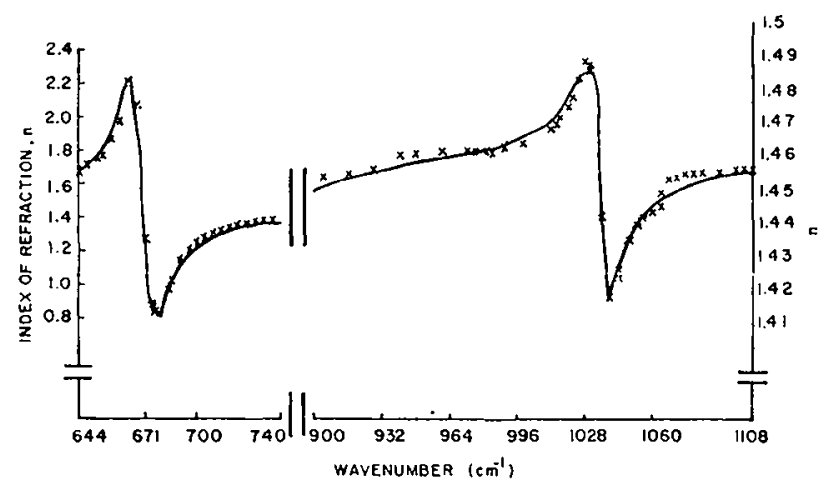

FIG. 2. - Refractive index of benzene in the spectral region of two main absorption bands. The solid lines were obtained by PK transformation of k obtained directly from figure $1 \mathrm{data}$. The crosses are values of $\mathbf{n}$ from reference [3]. of any transformation. In figure 3 is given $n(\omega)$ of benzene over the entire spectral range of our data on a single scale.

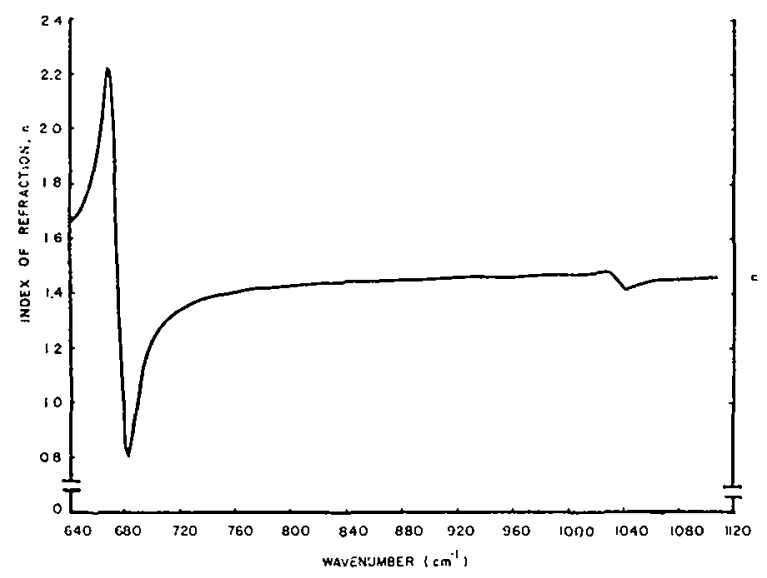

Frg. 3. - Refractive index of benzene over the entire spectral range, on the same scale to show relationships of the two bands.

It is interesting to note that tabulated transmission spectra such as those in Sadtler tables can be transformed to give $n(\omega)$ for any case of interest. This would be greatly facilitated if the spectra were available in digital form, ready for computer input. Of course the accuracy of the results would reflect that of the data.

3. Application to internal reflection. -- For maximum electromagnetic interaction with the small amount of material that constitutes the solid-liquid interphase, it is often advantageous to use internal reflection. The reason is that the mean square electric field, $\left\langle E^{2}\right\rangle$, can be made much larger than for the external reflection case. This is due mainly to the special properties of the evanescent wave [4]. Surprising as it might seem, thin metal layers can be used to enhance the $\left\langle E^{2}\right\rangle$ of the evanescent wave [5].

First consider the case of attenuated total reflection, ATR, where we wish to examine spectroscopically a thin layer on a transparent substrate. The substrate may comprise a number of transparent phases, homogeneous or continuously varying in the direction perpendicular to the interface, and the whole may be in contact with a liquid phase. An example might be a transparent prism supporting a metal oxide layer on which a material is adsorbed from aqueous electrolyte.

The general procedure is to use experimental data to tabulate $\operatorname{Re} G(\omega)=\frac{1}{2} \ln \left(R_{\mathrm{s}} / R_{\mathrm{b}}\right)$ throughout an absorption band or over an extended spectral range of interest. This is especially simple for ATR because $R_{\mathfrak{b}}$ is unity. A PK transformation of $\operatorname{Re} G(\omega)$ gives

$$
\operatorname{Im} G(\omega)=\left(\theta_{\mathrm{s}}-\theta_{\mathrm{b}}\right)
$$

everywhere. But $\theta_{b}$ is linear in the spectral region of interest [1]. Auxiliary knowledge of $\theta_{\mathrm{s}}$ at, say, two points gives $\theta_{b}$ at two points and throughout the region by extrapolation, which in turn gives $\theta_{s}$ throughout. 
$\left(\theta_{s}\right.$ at two points is calculated from known or determined optical properties at two points. These points may be outside the absorption band where determination is simple.) The values of $R_{\mathrm{s}}$ and $\theta_{\mathrm{s}}$ are then available to solve problems of interest concerning the system. In particular, if the sample thickness, $h$, is known $R_{\mathrm{s}}$ and $\theta_{\mathrm{s}}$ can be used to solve for $n_{\mathrm{s}}(\omega)$ and $k_{\mathrm{s}}(\omega)$ by means of numerical non-linear inversion techniques [6].

Linearized approximates equation have been derived for ATR [7, 8] and other cases of interest. They make the physics much clearer for multiphase systems. They are especially useful for analyzing very thin films represented by adsorption at a surface. The PK causal transformation can be applied in a very direct way to these equations to derive maximum information from ATR spectra.

Experimentally it is convenient to measure reflection spectra as the change in reflection absorbance $\left(A \equiv-\log _{10} R\right.$, and $\left.\Delta A \equiv \log _{10}\left(R_{\mathrm{b}} / R_{\mathrm{s}}\right)\right)$, caused by $k_{\mathrm{s}}$. For ATR at the critical angle of incidence, $\varphi=\varphi_{\mathrm{c}}$, the linear equation are, for $\perp$ polarization

$$
\Delta A_{\perp}=\frac{8 \pi}{\lambda \ln 10}\left(\frac{h_{2}}{n_{1} \cos \varphi_{1}}\right) \varepsilon^{\prime \prime} \quad\left(\varphi=\varphi_{\mathrm{c}}\right)
$$

and for || polarization

$$
\Delta A_{\|}=\frac{8 \pi}{\lambda \ln 10}\left(\frac{h_{2} n_{1}^{2} n_{3}^{2}}{n_{1} \cos \varphi_{1}}\right) \frac{\varepsilon^{\prime \prime}}{\varepsilon \varepsilon^{*}} \quad\left(\varphi=\varphi_{\mathrm{c}}\right) .
$$

Here subscripts 1, 2 and 3 refer to the incident, second and final phases respectively, $h$ is the thickness of the second phase, and $\varepsilon \equiv \varepsilon^{\prime}+i \varepsilon^{\prime \prime}$ is the complex dielectric constant of the second phase. Note that $\left(\varepsilon^{\prime \prime} / \varepsilon \varepsilon^{*}\right)=\operatorname{Im}\left(1 / \varepsilon^{*}\right)$, the dielectric loss function. It is well known that $\varepsilon(\omega)-1$ is causal [9]. It can also easily be shown that $\left(\left(1 / \varepsilon^{*}\right)-1\right)$ is causal. Recall also that the sum of two causal functions is causal.

Now to solve for the intrinsic spectra, i. e., $\varepsilon(\omega)$ or $n(\omega)$, from experimental $\Delta A_{\perp}$ and $h_{2}$ values, proceed as follows. Solve for $\varepsilon^{\prime \prime}$ using eq. (9). This is also the value of $\varepsilon^{\prime \prime}-\varepsilon_{\mathrm{b}}^{\prime \prime}$ since $\varepsilon_{\mathrm{b}}^{\prime \prime}$ is essentially zero in the region of data. Now PK transform to $\varepsilon^{\prime}-\varepsilon_{\mathfrak{b}}^{\prime}$. Use auxiliary information for $\varepsilon^{\prime}$ at one or two frequencies to solve for linear $\varepsilon_{\mathrm{b}}^{\prime}$. Now $\varepsilon^{\prime}$ is known throughout the region of data. Then $\varepsilon=\widehat{n}^{2}$ gives $\widehat{n}(\omega)$.

Parallel polarization usually gives the high $\left\langle E^{2}\right\rangle$ and therefore high sensitivity. The values of $\left(1 / \varepsilon^{*}\right)$ can be obtained from $\Delta A_{\|}$by exactly the same procedures used with $\varepsilon$. Of course $\varepsilon$ is easily obtained from the determined $\left(1 / \varepsilon^{*}\right)$, and likewise for $\hat{n}$.

An interesting point is that this method provides a means of determining the optical constants (intrinsic spectra) of an anisotropic thin film. For very thin films at $\varphi=\varphi_{c}$, parallel polarization gives an electromagnetic field perpendicular to the plane of the film. Thus the dielectric constant perpendicular to the film, $\varepsilon_{\perp}$, is determined. Using perpendicular polarization the constant for any direction in the plane of the film is determined. This completely determines the anisotropic dielectric constant for a film or uniaxial symmetry. If the plane of the interface can be rotated with respect to the beam, even lower symmetry can be analyzed by using the $\Delta A_{\perp}$ equation repeatedly. Of course for $\perp$ polarization the electric field is always in the plane of the interface regardless of angle of incidence.

The linear equations for angles of incidence other than $\varphi_{\mathbf{c}}$ are only slightly more complicated [7]

$$
\begin{gathered}
\Delta A=\frac{8 \pi h_{2}}{\lambda \ln 10}\left(\frac{n_{1} \cos \varphi_{1}}{n_{1}^{2}-n_{3}^{2}}\right) \varepsilon^{\prime \prime} \\
\Delta A_{\|}=\frac{8 \pi h_{2}}{\lambda \ln 10}\left(\frac{\eta_{1}}{\eta_{1}-\eta_{3}}\right) \times \\
\times\left(\frac{n_{1}^{2} \sin ^{2} \varphi_{1}}{\varepsilon \varepsilon^{*}}-\eta_{3}^{2}\right) \varepsilon^{\prime \prime}
\end{gathered}
$$

where $\eta_{j} \equiv \cos \varphi_{j} / n_{j}$. The first equation is handled as before, leading to $\varepsilon^{\prime}$, hence to $\varepsilon(\omega)$. The second is slightly more complicated. We can write it in the form $\Delta A_{\|}=c \operatorname{Im}\left(a / \varepsilon^{*}+b \varepsilon\right)$. The quantity in parentheses minus $(a+b)$ is causal. It is $\mathrm{PK}$ transformed and otherwise treated as described above. The result is a complete determination of the complex quantity $\left(a / \varepsilon^{*}+b \varepsilon\right)$ in the spectral range of interest. Since $a$ and $b$ are known constants $\varepsilon(\omega)$ and $\hat{n}(\omega)$ are determined.

The approximate linear equations apply for much more complicated situations where multiple layers are allowed including metal layers $[5,7,8]$. Similar transformations apply. This transformation method can be applied in like fashion to the linearized equations of J. D. E. McIntyre [8] used in his demetallization effects and surface layer absorption.

Of course the general procedures outlined at the beginning of this section are always applicable. The use of the linearized equations is more direct, however, and avoids the nonlinear inversion of $G(\omega)$ to get $\varepsilon(\omega)$. Where they apply, they also give more physical insight at each step.

4. Application to external reflection. - The general procedures for treating external reflection spectra are the same as outlined in section 3 . In some cases, however, it may be more difficult to derive $\ln \left(R_{\mathrm{s}} / R_{\mathrm{b}}\right)$ from the measured reflectance. For many cases this is no problem. These occur when the reflectance is not sensitive to the refractive index of the bleached sample layer. A thin absorbing film on a highly reflecting bulk metal surface is such a case. An example is an oxide coated metal, or a similar system with an additional absorbing adsorbed organic film to give a four phase system. Another example is a solid-liquid system with a layer at the interface whose refractive index when the sample is bleached is near that of the liquid phase (or solid phase), or with a layer so thin that to first order it has no effect on the reflectance when bleached. (Recall 
that the phase angle change on reflection is much more affected by thin transparent layers than is reflectance. This gives ellipsometry its high sensitivity.)

Again linearized approximate equations can be very useful. They give the physical insight necessary to choose experimental parameters wisely and point the way to optimum data utilization. To illustrate procedures and utility we will discuss in detail the case of a thin film, or films, on a highly reflecting metal substrate.

First order equations for an absorbing layer on a highly reflecting metal surface are

$$
\Delta A_{\|}=\left(\frac{8 \pi h n_{1}^{3} \sin ^{2} \varphi_{1}}{2.3 \lambda \cos \varphi_{1}}\right) \frac{\varepsilon^{\prime \prime}}{\varepsilon \varepsilon^{*}}
$$

and

$$
\Delta A_{\perp}=\left(\frac{8 \pi h \cos \varphi_{1}}{2.3 \hat{\lambda} n_{1} k_{3}^{2}}\right) \varepsilon^{\prime \prime}
$$

where $\varepsilon$ represents the layer sought (the subscript « $s$ » has been omitted), $h$ is the unknown layer thickness, and $k_{3}$ is $\operatorname{Im} \hat{n}$ of the metal substrate. These equations were derived and discussed in reference [7]. A number of important points become clear immediately. First of all, it is necessary to use parallel polarization for high sensitivity. An absorbing molecule near the metal surface is essentially hidden if normal incidence or perpendicular polarization are used because $\left\langle E^{2}\right\rangle$ is very low. For a typical metal in the infrared $k_{3}^{2}$ is of the order of 1000 . This is illustrated by figure 4 . $\Delta A_{\perp}$ cannot even be seen on the scale given. A second point is that $\triangle A \equiv A_{\mathrm{s}}-A_{\mathrm{b}}$ is the same as $\Delta A^{\prime} \equiv A_{\mathrm{s}}-A_{\mathrm{ns}}$ (ns means no sample) because $A_{\mathrm{b}}=A_{\text {ns }}=0$. A third

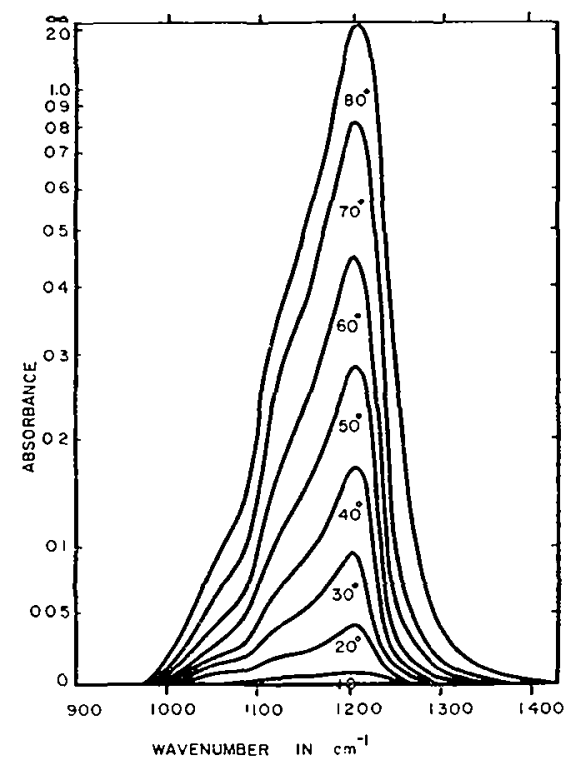

Fig. 4. - External refiection spectrum of silicon oxide on aluminum in the infrared. The oxide is a $0.1 \mu$ thick protective layer. Parallel polarization was used at the angles of incidence indicated. point is that the shape of the spectra should not change with angle of incidence.

In figure 4 are given infrared reflection spectra of a $0.1 \mu$ thick silicon oxide layer on an aluminum mirror. Note that the shape remains invariant even when most of the energy is absorbed. It is remarkable that at the peak of the $80^{\circ}$ curve only about one thousandth of the energy remains. The base line was determined by extrapolation from regions on both sides of the band where there is no absorption. Thus the spectra give

$$
\Delta A_{\|}=A_{\| \mathrm{s}}-A_{\| \mathrm{b}}
$$

directly with no approximation, where $A_{\| \mathrm{b}}$ refers to reflection absorbance from the metal plus layer system with the sample electromagnetically bleached. In fact a transparent thin film cannot be seen in the present experiment. The intrinsic spectra of the silicon oxide film were obtained by a PK transformation of $\left(\varepsilon^{\prime \prime} / \varepsilon \varepsilon^{*}\right)_{\mathrm{s}}-\left(\varepsilon^{\prime \prime} / \varepsilon \varepsilon^{*}\right)_{\mathrm{b}}$ which was calculated from the measured $\Delta A_{\|}$using eq. (13). $\left(\varepsilon^{\prime \prime} / \varepsilon \varepsilon^{*}\right)_{b}$ is of course essentially zero in the region of the spectrum and equal to $\left(\varepsilon^{\prime \prime} / \varepsilon \varepsilon^{*}\right)_{s}$ everywhere else. Thus the PK transformation gives $\left(\varepsilon^{\prime} / \varepsilon \varepsilon^{*}\right)_{\mathrm{s}}-\left(\varepsilon^{\prime} / \varepsilon \varepsilon^{*}\right)_{\mathrm{b}}$. The term $\left(\varepsilon^{\prime} / \varepsilon \varepsilon^{*}\right)_{\mathrm{b}}$ is by no means zero and must be determined from minor auxiliary information as discussed in the section on internal reflection. We now have both $\operatorname{Im}\left(1 / \varepsilon^{*}\right)$ and $\operatorname{Re}\left(1 / \varepsilon^{*}\right)$, i. e., we have $\left(1 / \varepsilon^{*}\right)$ from which $\varepsilon$ is immediately available as well as $\hat{n}$. Thus we have obtained $n(\omega)$ and $k(\omega)$ throughout the absorption band. They completely describe the silicon oxide layer in this spectral region in the ordinary optical sense. This procedure has been carried out for the film of figure 4 and the resulting $\hat{n}(\omega)$ found to have typical values.

The spectra of figure 4 are proportional to and therefore shaped like $\operatorname{Im}\left(1 / \varepsilon^{*}\right)$. They differ markedly from the intrinsic spectra $n(\omega)$ and $k(\omega)$ or $\varepsilon^{\prime}(\omega)$ and $\varepsilon^{\prime \prime}(\omega)$ calculated from them. It is hard to see how the intrinsic spectra could be obtained other than by the methods presented here. Spectral scanning ellipsometry could be used, but it is a difficult technique at these frequencies in the infrared. It might be thought that $n$ and $k$ could be obtained from data at two different angles of incidence. Eq. (13) shows why this is not a sensitive procedure. Two equations at different $\varphi$, cannot be inverted to give independent equations for $\varepsilon^{\prime}$ and $\varepsilon^{\prime \prime}$, or $n$ and $k$.

5. Discussion. - The detailed application of our method to selected cases has been made to clarify procedures and to demonstrate their utility. The heart of the method is the concept of electromagnetic bleaching which eliminates absorption bands in the spectral range of interest. For our purposes this is only a thought process, of course, although bleaching is an observed natural phenomenon.

The procedures can be applied to reflection and transmission cases other than the ones discussed. Any specular reflectance can be transformed if a quantity equivalent to $\left(R_{\mathrm{s}} / R_{\mathrm{b}}\right)$ can be measured throughout 
an absorbing range. Also the more direct procedure of transforming linearized reflection and transmission equations can be extended to other cases discussed in references [7] and [8]. Reflection from less reflecting metals such as mercury, gold, and platinum in the visible spectral range can be analyzed similarly. Our procedures are especially clear for isolated absorption bands or groups of bands. They are also applicable to nonisolated absorption if the spectral range of experimental data is wide enough so that edge effects are unimportant to a central region of interest. We have not treated here the case of a metallic sample with its free electron absorption.

The type of spectral modulation where no new material is added or removed, but spectral changes occur because of electronic changes can be thought of as a change analogous to partial bleaching. Here the process is not just imagined, but actually occurs. Metallic electroreflectance is an example. So is spectral change at electrodes caused by adsorption, to the extent that the change is due to changes in the electrode rather than due to the intrinsic properties of the adsorbate. This case of spectral modulation is directly transformable to give $\Delta \theta$ from $\Delta(\ln R)$ provided the latter is known throughout the spectral range of the effect. It doesn't matter in this case that the sample is the metal surface itself. The complexity of the interphase is also unimportant to this transformation. It may however, be very important to interpretations of the results. It is assumed in this discussion of spectral modulation that the changes in spectra are confined to a reasonable spectral width where measurements can be made, i. e., contributions to the PK transformation from regions outside the data range can be neglected.

Appendix A. - Here we will derive eq. (4) and (5) from (2) and (3) which were taken from the paper of Peterson and Knight [2]. We will use the PK notation with minor changes. Note that their eq. $(10 c)$ has an error. The next to the last $\exp (i \omega t)$ should be changed to $\exp (-i \omega t)$.

From the PK paper

$$
\begin{aligned}
I(t) & \left.=\frac{1}{2 \pi} \int_{-\infty}^{\infty} F(\omega) \mathrm{e}^{i \omega t} \mathrm{~d} \omega \quad \text { (PK eq. }(2 a)\right) \\
& =\frac{1}{2 \pi}\left[\int_{-\infty}^{\infty} S(\omega)(\cos \omega t+i \sin \omega t) \mathrm{d} \omega+\int_{-\infty}^{\infty} i A(\omega)(\cos \omega t+i \sin \omega t) \mathrm{d} \omega\right]
\end{aligned}
$$

where

$$
F(\omega)=S(\omega)+i A(\omega) \quad(P K \text { eq. }(5))
$$

and $S(\omega)$ is symmetric and $A(\omega)$ antisymmetric.

By symmetry

$$
\begin{aligned}
I(t) & =\frac{1}{2 \pi} \int_{-\infty}^{\infty} S(\omega) \cos \omega t \mathrm{~d} \omega-\frac{1}{2 \pi} \int_{-\infty}^{\infty} A(\omega) \sin \omega t \mathrm{~d} \omega \\
& =I_{\mathrm{S}}(t)+I_{\mathrm{A}}(t) .
\end{aligned}
$$

For $t<0, I(t)=0$ and $I_{\mathrm{A}}(t)=-I_{\mathrm{S}}(t)$. Therefore, for $t>0, I_{\mathrm{A}}(t)=I_{\mathrm{S}}(t)$ and

$$
\frac{1}{2 \pi} \int_{-\infty}^{\infty} S(\omega) \cos \omega t \mathrm{~d} \omega=-\frac{1}{2 \pi} \int_{-\infty}^{\infty} A(\omega) \sin \omega t \mathrm{~d} \omega
$$

taking the cosine transform of both sides gives

$$
S\left(\omega_{0}\right)=-\frac{1}{2 \pi} \int_{-\infty}^{\infty} \mathrm{d} t \cos \omega_{0} t \int_{-\infty}^{\infty} \mathrm{d} \omega A(\omega) \sin \omega t
$$

taking the sine transform of (A6) gives

$$
A\left(\omega_{0}\right)=-\frac{1}{2 \pi} \int_{-\infty}^{\infty} \mathrm{d} t \sin \omega_{0} t \int_{-\infty}^{\infty} \mathrm{d} \omega S(\omega) \cos \omega t
$$

and finally from (A7) and (A8) by symmetry,

$$
S\left(\omega_{0}\right)=-\frac{2}{\pi} \int_{0}^{\infty} \int_{0}^{\infty} A(\omega) \sin \omega t \cos \omega_{0} t \mathrm{~d} \omega \mathrm{d} t
$$

and

$$
A\left(\omega_{0}\right)=-\frac{2}{\pi} \int_{0}^{\infty} \int_{0}^{\infty} S(\omega) \cos \omega t \sin \omega_{0} t \mathrm{~d} \omega \mathrm{d} t
$$


Appendix B. -- For complete analysis of reflection or transmission spectra by means of causal transformation it is necessary to know the optical constants and thickness of the sample film at one or two frequencies within the spectral range used. One approach is to guess $\hat{n}$ from known values in other spectral regions or from the known properties of similar substances. It is often possible to avoid these uncertain procedures by direct measurement. This is the approach we favor and discuss below.

Many powerful techniques have been developed to characterize thin films as to optical constants and thickness. Reviews are given by F. Abelés [10] and by H. E. and J. M. Bennett [11]. We have found convenient the direct optical determination of $\hat{n}$ and $h$ by inversion of multiple reflection, transmission, and/or ellipsometric measurements [12, 13].

This latter approach succeeds sometimes when other methods fail. For example, from the data of figure 1 reference [7] which comprises four independent reflection spectra of a crystal violet monolayer on glass, it was found by numerical inversion that the thickness was $22.5 \AA$. Thickness and $\hat{n}$ could be determined with high accuracy at a number of frequencies from the data, but the complete intrinsic spectra, $n(\omega)$ and $k(\omega)$ were best determined using a PK transformation of one of the reflection curves and making use of the known $\hat{n}$ and $h$ values.
In the case of an oxide layer on a metal to be studied in the infrared, the index of the bleached sample can be estimated from literature values. Better yet, it can be determined along with its thickness by ellipsometry in the visible and extrapolated to the infrared region of interest.

For samples thicker than a few microns, a simple refractometer can be used to determine refractive index. Using a variable wavelength source, $n$ can be readily determined at a number of wavelengths making extrapolation easy. Measurements with a standard refractometer need not be limited to the visible. Using a viewing device sensitive to the infrared, the spectral range can be greatly extended. A procedure handy for determining $n$ for liquids and solids at any wavelength is the variable angle reflection device discussed in reference [12]. It can be used in a simple infrared spectrophotometer, for example, to determine $n_{\mathrm{s}}(\omega)$ by locating the critical angle between sample and prism. By this means accuracies of better than one percent are routinely obtained on a Beckman IR-8.

Acknowledgments. - Acknowledgment is made to the Donors of the Petroleum Research Fund, administered by the American Chemical Society, for partial support of this research.

\section{References}

[1] Hansen, W. N. and Wedad ABdou, Causality Calculations in Reflection Spectroscopy to be published in, J. Opt. Soc. Am.

[2] Peterson, C. W. and Knight, B. W., J. Opt. Soc. Am. 63 (1973) 238.

[3] Gilby, A. C., Burr, J. Jr., Krueger, W. and Crawford, B., J. Phys. Chem. 70 (1966) 1525.

[4] Hansen, W. N., J. Opt. Soc. Am. 58 (1968) 380.

[5] HaNSEN, W. N., Internal Reflection Spectroscopy, in Advances in Electrochemistry and Electrochemical Engineering, P. Delahay and C. W. Tobias eds. (John Wiley, New York) 1973, p. 25.

[6] Bevington, P. R., Data Reduction and Error Analysis for the Physical Sciences (McGraw-Hill, New York) 1969.

[7] Hansen, W. N., Reflection Spectroscopy of Adsorbed Layers Symposium of the Faraday Socicty 4 (1970) 27.
[8] MCINTYRe, J. D. E., Optical Reflection Spectroscopy of Chemisorbed Monolayers in Optical Properties of Solids New Developments B. O. Seraphin ed. (American Elsevier Publishing Co. New York) 1976, Chapter 11.

[9] LANDAU, L. D. and LifSHiTz, E. M., Electrodynamics of Continuous Media (Pergamon Press, New York) 1960, Section 62.

[10] Abelés, F. in Physics of Thin Films 6, G. Hass and R. E. Thun eds. (Academic Press, New York) 1971, pp. 151-204.

[11] Bennett, H. E. and Bennett, J. M., in Physics of Thin Films 4, G. Hass and R. E. Thun eds. (Academic Press, New York) 1967, pp. 1-96.

[12] Hansen, W. N., Anal. Chem. 37 (1965) 1142.

[13] Anderson, W. J. and Hansen, W. N., Optical Characterization of Thin Films, J. Opt. Soc. Am. 67 (1977) 1051.

\section{DISCUSSION}

W. PLieth. - In general organic molecules have a prefered orientation on a metal surface and one should expect anisotropy of the optical properties. The calculation of the phase shift has the great advantage that you can determine this anisotropy. Do your measurements and calculations give any information regarding the structure of your films?

W. HANSEN. - Our methods are well suited to the study of thin film anisotropy. If parallel polarization internal reflection spectra are taken at the critical angle, for example, the electromagnetic electric fields in the film are normal to the surface, and the optical constants normal to the surface are determined. On the other hand, if perpendicular polarization is used, the optical constants in the plane of the film will be determined regardless of angle of incidence. Thus for common uniaxial anisotropy, both sets of constants can be determined.

For crystal violet layers on glass and on gold, the 
films appear to be nearly isotropic, although this information was not specifically sought when the data were taken, and the angle of incidence is somewhat greater than critical. Surface roughness may also play a role.

D. LYNCH. - How does your method differ, other than in the numerical method used, from a KramersKronig analysis of a differential reflectance spectrum ? In such a case neglected structure outside the range of the measured data plays a far smaller role than it does in the KK analysis of the reflectance itself (see, e. g., Balzarotti, A., Colavita, E., Gentile, S. and Rosef, R., Appl. Opt. 14 (1975 2412). Once the reflectance of a complicated manylayered material has been KK analyzed to give the phase, the further analysis, e. g. to obtain $\varepsilon$ for the $n^{\text {th }}$ layer, all other $\varepsilon^{\prime}$ s being known, is an algebraic problem.

W. HANSEN. - It is by no means obvious that general reflection spectra from stratified media can be subjected to transformations of the KK or PK type and analyzed by procedures commonly used for twophase systems. In fact such analysis is not generally valid. Even the addition of a simple transparent window to the two-phase case introduces surprising complexities discussed by Plaskett and Schatz [1]. The three-phase case is discussed by Plieth and Naegele $[2,3]$. They showed that for very thin films $\ln \left(\hat{r}_{123} / \hat{r}_{13}\right)$ can be transformed. (Here, $\hat{r}_{123}$ is for the film-covered surface and $\hat{r}_{13}$ is for the uncovered surface). They found that, due to possible singularities, a Blashke

[1] Plaskett, J. S. and Schatz, P. N., J. Chem. Phys. 38 (1963) 612.

[2] Plieth, W. J. and Naegele, K., Surf. Sci. 50 (1975) 53.

[3] Naegele, K. and Plieth, W. J., Surf. Sci. 50 (1975) 64 product may have to be added to the usual transform integral even in this case. These systems, while much more complex than the common two-phase case, are very simple compared to the general stratified medium addressed by the present approach.

R. ADzIC. - The wavelength dependance of $\Delta R / R$ of gold in the presence of $\mathrm{I}^{-}$you have shown differs in certain detail from the spectrum we have obtained by external reflectance measurements, that spectrum shows an additional peak at $540 \mathrm{~nm}$. Two peaks, although at different wavelengths, have been found also by Lazarenko-Manevich et al.

Can you comment on these differences ?

W. HANSEN. - Our electroreflectance spectra were obtained by means of internal reflection using perpendicular polarization at greater than critical angle. The light samples the entire film of gold and its surface region. Thus, for example, a change in thickness of the gold would be seen. This response differs fundamentally from the external reflection case. In addition the films are prepared by evaporation onto glass and differ somewhat from bulk gold.

K. NAEGELE. - Your experiments are restricted to a limited photon energy range. How did you check that you have no influence on your results from other spectral regions? Is it possible that this influence not taken into account produces the observed and surprising isotropic behaviour of the thin adsorbed film ?

W. HANSEN. - Our procedure are designed to give accurate results if experimental data are available throughout an absorption band or group of bands. Very little error should have been introduced by the limited photon energy range because the range included nearly a complete band in refiectance in the case of the cristal violet spectra. 